\title{
In vitro cultivation of Babesia duncani (Apicomplexa: Babesiidae), a zoonotic hemoprotozoan, using infected blood from Syrian hamsters (Mesocricetus auratus)
}

\author{
Kimberly A. McCormack ${ }^{1,2}$ (D) $\cdot$ Amer Alhaboubi $^{3,4} \cdot$ Dana A. Pollard $^{3,5} \cdot$ Lee Fuller $^{6}$ - Patricia J. Holman ${ }^{2}$
}

Received: 16 November 2018 / Accepted: 5 June 2019 / Published online: 13 June 2019

(C) Springer-Verlag GmbH Germany, part of Springer Nature 2019

\begin{abstract}
Human babesiosis, a tick-borne disease similar to malaria, is most often caused by the hemoprotozoans Babesia divergens in Europe, and Babesia microti and Babesia duncani in North America. Babesia microti is the best documented and causes more cases of human babesiosis annually than all other agents combined. Although the agents that cause human babesiosis are considered high-risk pathogens in transfusion medicine, federally licensed diagnostics are lacking for $B$. duncani in both the USA and Canada. Thus, there has been a need to develop and validate diagnostics specifically for this pathogen. In this study, B. duncani (WA1 isolate) was cultivated in vitro from Syrian hamster (Mesocricetus auratus) infected blood. We hypothesized HL-1 media with supplements would result in B. duncani propagating at higher levels in culture than supplemented M199 similar to the medium the parasite was originally cultivated with in 1994. We were unable to recreate Thomford's cultivation results with the M199 medium but supplemented HL-1 medium was able to successfully establish continuous culture. We further hypothesized that RBC from species other than hamsters would support $B$. duncani in vitro. However, rat, mouse, horse, and cow RBC did not support continuous culture of the parasite. Culture stocks of $B$. duncani were deposited at BEI Resources and are now commercially available to the scientific community to further research. The cultured parasite developed in this study was instrumental in the adaptation of $B$. duncani continuous culture to human RBC.
\end{abstract}

Keywords Babesia duncani $\cdot$ Human babesiosis $\cdot$ Transfusion $\cdot$ Tick-borne disease $\cdot$ Percentage of parasitized erythrocytes (PPE)

\section{Introduction}

Human babesiosis is a tick-borne disease similar to malaria caused by intraerythrocytic protozoan parasites. There are a number of recognized zoonotic Babesia spp. in North America at present, among which Babesia microti and Babesia duncani (Conrad et al. 2006) cause the most cases

Section Editor: Leonhard Schnittger

Kimberly A. McCormack

Kim.McCormack@okstate.edu

1 Comparative Medicine Program, Department of Veterinary Pathobiology, College of Veterinary Medicine and Biomedical Sciences, Texas A\&M University, College Station, TX 77843-4473, USA

2 Present address: Department of Comparative Medicine, University of Oklahoma Health Sciences Center, 940 Stanton L. Young Boulevard Room 203, Oklahoma City, OK 73104, USA of human babesiosis and thus are the most clinically relevant (Conrad et al. 2006). B. duncani was initially described in Washington as WA1 and California as CA5 isolates with an unknown tick vector and animal host. The search for animal hosts led researchers to discover mice, gerbils, and hamsters are susceptible to experimental infection with $B$. duncani (Conrad et al. 2006) (Kjemtrup and Conrad 2000). However,
3 Department of Veterinary Pathobiology, College of Veterinary Medicine and Biomedical Sciences, Texas A\&M University, College Station, TX 77843-4467, USA

4 Present address: Department of Parasitology, College of Veterinary Medicine, University of Baghdad, Baghdad 10001-10090, Iraq

5 Present address: Health Resources and Services Administration, U.S. Department of Health and Human Services, 5 Skip Bertman Drive, Baton Rouge, LA 70803, USA

6 Fuller Laboratories, 1312 East Valencia Drive, Fullerton, CA 92831, USA 
recent evidence suggests Dermacentor albipictus (Packard, 1869) (winter tick) and Odocoileus hemionus (Rafinesque, 1817) (mule deer) may fulfill these roles (O'Connor et al. 2018; Swei et al. 2019). If so, the geographical range of $B$. duncani could be expected to extend to the overlapping regions of this potential tick vector and reservoir host species across the USA and Canada. Mule deer are found throughout western North America from southern Canada to north of central Mexico and as far east as Minnesota and Iowa in the USA (Innes 2013). The winter tick is found throughout the USA and ranges from its southern-most border in Central America to as far north as Alaska (TAMUS 2011).

Cases of human babesiosis in the USA have been increasing over the last two decades. In 2011, babesiosis was added to the list of nationally notifiable diseases maintained by the Centers for Disease Control and Prevention (CDC) (Herwaldt et al. 2012). Human babesiosis has been reportable in 27 states since 2013, with $95 \%$ of cases occurring in Connecticut, Massachusetts, Minnesota, New Jersey, New York, Rhode Island, and Wisconsin (Moritz et al. 2016). Babesiosis is most commonly acquired from a tick bite. Many infected individuals are asymptomatic. Those who present with the disease have signs ranging from mild flu-like to severe malaria-like symptoms (Herwaldt et al. 2012). It can be appropriately treated when correctly diagnosed and usually resolves without complication in immunocompetent patients. The parasite can easily be transmitted through blood transfusions and cases of transfusion-transmitted babesiosis tend to be more severe or even lethal (Lobo et al. 2013). Immunocompromised individuals, especially those receiving multiple blood transfusions, are more at risk for acquiring and developing the severe lifethreatening disease. United States Food and Drug Administration (USFDA) statistics from 2008 to 2012 show that babesiosis was responsible for the highest percentage of deaths due to microbial infection resulting from blood transfusion (USFDA 2016). The CDC data archives show there were seven cases of transfusion-associated babesiosis reported in 2012, 14 cases in 2013, and six cases for 2014 (CDC 2015, 2016; Herwaldt et al. 2012; Herwaldt and Gray 2016). FDA statistics show two patients died during 2016 after receiving blood transfusions likely contaminated with Babesia (USFDA 2017).

The first report to date of $B$. duncani (WA1) continuous in vitro culture and the initial characterization was in 1994 (Thomford et al. 1994). Whole infected human blood from a patient was passaged through a Syrian hamster twice. Thomford et al. then initiated cultures in M199 supplemented with FBS using blood from a low parasitemic hamster $(3.5 \%)$. Actively growing parasites were subcultured into hamster RBC every 2-3 days. Thomford et al. cryopreserved and resuscitated culturederived piroplasms and showed the pathogenicity was maintained through hamster inoculation.
In the current study, blood was collected from highly parasitemic hamsters $(10-13 \%)$. Cultures were subcultured every 2-7 days when blood smears showed elevated PPE (percentage of parasitized erythrocytes). Our study aimed to compare the growth of $B$. duncani in HL-1 supplemented versus M199 supplemented medium, and to compare the initiation of cultures directly from blood cryostock versus blood from hamsters with active infection. We further hypothesized that RBC from species other than hamsters will be able to successfully maintain $B$. duncani in continuous culture and mouse, rat, cow, and horse RBC were tested.

\section{Materials and methods}

\section{In vivo}

\section{Animals}

Adult male Syrian hamsters $(n=10,>100 \mathrm{~g})$ (Envigo RMS, Inc., Haslet, MI) were pair housed in standard laboratory rat cages (XL Allentown Static Rodent Cage) at Texas A\&M University and were fed standard rodent diet (Envigo Teklad Rodent Diet 8604) and water ad libitum. All housing and procedures were performed in facilities accredited by the Association for Assessment and Accreditation of Laboratory Animal Care (AAALAC) and conducted in accordance with Texas A\&M University Institutional Biosafety Permit (IBC 2012-133) and approved by the Texas A\&M Institutional Animal Care and Use Committee (IACUC) (AUP 20150256).

\section{Donor blood}

Hamster blood for donor RBC was either obtained from individual hamsters housed at Texas A\&M University (TAMU hamster) or as a pooled lot from a commercial source (BioChemed Services, Winchester, VA). In both cases, blood was collected into EDTA K2. For hamsters housed at Texas A\&M University, the animals were anesthetized with isoflurane for retro-orbital venipuncture. A safe amount of whole blood $(0.5-1.7 \mathrm{ml})$ depending on hamster size was collected into $1.3 \mathrm{ml} \mathrm{K} 3$ EDTA microtubes (Sarstedt \#41.1395.105). At least 2 weeks rest was given between venipuncture events and collection was from alternate eyes. Donor RBC from cow, horse, rat, and mouse were obtained through the Tissue Sharing program at Texas A\&M University that allows the use of extra blood procured through other IACUC approved protocols.

All blood was centrifuged at $870 \times \mathrm{g}$ for $3 \mathrm{~min}$ in a microfuge to pellet the cells, then the plasma and buffy coat were removed and discarded. The cells were washed 3 times by centrifugation in at least 5 volumes of Puck's 
Saline Glucose (PSG) (Alfa Aesar \#J67542) with careful removal of the supernatant and buffy coat at each wash. The washed RBC were stored in an equal volume of PSG plus extra glucose $(20 \mathrm{~g}$ glucose/L) with a final concentration of $200 \mu \mathrm{g} / \mathrm{ml}$ streptomycin, $200 \mathrm{U} / \mathrm{ml}$ penicillin, and $50 \mu \mathrm{g} / \mathrm{ml}$ Amphotericin B (Antibiotic/Antimycotic, Atlanta Biologicals \#022110) at $4{ }^{\circ} \mathrm{C}$ for a maximum of 2 weeks.

\section{Hamsters inoculated for culture}

Babesia duncani was procured as cryopreserved WA1 infected hamster blood (NR-12311) from BEI Resources (National Institute of Allergy and Infectious Diseases, Manassas, VA). Upon receipt, the vial containing the cryopreserved $B$. duncani was swirled in a $37^{\circ} \mathrm{C}$ water bath until the contents were thawed, then the entire contents $(\sim 0.5 \mathrm{ml})$ were inoculated intraperitoneally (i.p.) into a Syrian hamster. Day of inoculation was considered day 0 . The inoculated hamster was observed daily for any clinical signs. The parasitemia was monitored daily by microscopic examination of blood smears stained with Giemsa at $\times 1000$ under oil immersion. Blood samples for the smears were obtained through lateral saphenous venipuncture and alternating sites were used each day. At 10 days, when the parasitemia was approximately $13 \%$, the hamster was anesthetized with isoflurane and blood collected via terminal cardiac puncture into EDTA and $\sim 0.5 \mathrm{ml}$ of the infected blood was inoculated i.p. into hamster 2 (Fig. 1) as described in the WA1 information sheet for in vivo passaging. Part of the collected blood was used to initiate $B$. duncani cultures as below, and part was cryopreserved as below. The second hamster was anesthetized and blood collected via cardiac puncture into EDTA (Fig. 1) 3 days after inoculation when the parasitemia was approximately $10 \%$. Part of the blood was used for culture as below and part was cryopreserved in $0.5 \mathrm{ml}$ aliquots.

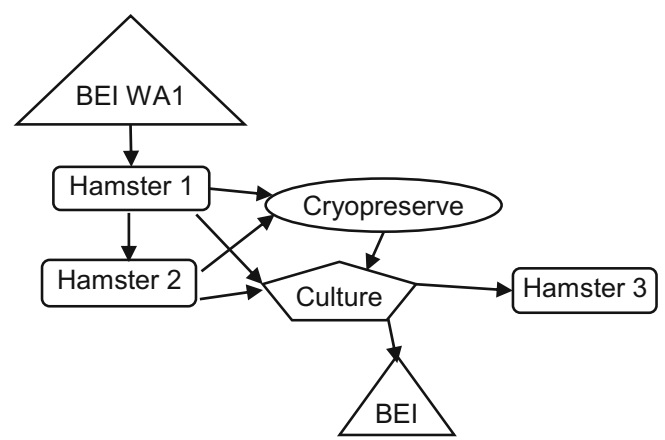

Fig. 1 Flowchart of B. duncani (WA1) in vivo passages through Syrian hamsters (Mesocricetus auratus) and in vitro cultures

\section{Hamster inoculated to test the infectivity of cultured B. duncani}

Cryopreserved passage 25 (P25) B. duncani culture (0.5 ml) that had been stored in liquid nitrogen for 34 days was thawed rapidly in a $37{ }^{\circ} \mathrm{C}$ water bath and inoculated i.p. into hamster 3. Hamster 3 was monitored daily for the appearance of infected $\mathrm{RBC}$ as described above.

\section{In vitro}

\section{B. duncani cultures initiated from BEl cryostock in $\mathrm{HL}-1$ and M199 media}

Cultures were initiated directly from cryopreserved B. duncani WA1 infected hamster blood (BEI \#NR12311). The vial containing the frozen infected hamster blood was swirled in a $37^{\circ} \mathrm{C}$ water bath until the contents were thawed. Immediately thereafter, $225 \mu 1$ was dispensed into two culture wells in a 24-well plate (SigmaAldrich \#3524) that each contained $70 \mu 1$ normal hamster donor RBC (BioChemed Services) and $900 \mu$ of media. The medium in one well consisted of HL-1 medium (Lonza \#77201) supplemented with 20\% fetal bovine serum (FBS) (Atlanta Biologicals \#S11550), $1 \mathrm{mg} / \mathrm{ml}$ AlbuMAX I ${ }^{\circledR}$ (Invitrogen \#11020-021), 2 mM -glutamine (Atlanta Biologicals \#B90210), 2\% HB101 ( $v / v$, reconstituted according to manufacturer's specifications; Irvine Scientific \#T151), $0.2 \mathrm{mM}$ sodium hypoxanthine and $0.032 \mathrm{mM}$ thymidine (Hypoxanthine-Thymidine; Sigma-Aldrich \#H0137-10VL), and a final concentration of $200 \mu \mathrm{g} / \mathrm{ml}$ streptomycin, $200 \mathrm{U} / \mathrm{ml}$ penicillin, and $50 \mu \mathrm{g} / \mathrm{ml}$ amphotericin B (Antibiotic/Antimycotic, Atlanta Biologicals \#022110). The second well contained medium 199 (M199) with Earle's balanced salt solution (Lonza \#12-119F) supplemented with 40\% FBS, 2 mM glutamine, $20 \mathrm{mM}$ TES (2- [Tris(hydroxymethyl)methylamino]-ethanesulfonic acid) (TCI America \#T0683), and the same antibiotic/antimycotic concentrations (Thomford et al. 1994). Each medium was filter sterilized (ThermoScientific Nalgene \#165-0045) after all components were added.

For all cultures, the culture medium was replenished daily by removing $800 \mu \mathrm{l}$ of the spent medium above the settled RBC layer and replacing with an equal volume of fresh medium. RBC smears were made daily from each culture well, stained with Giemsa, and examined at $\times$ 1000 under oil immersion microscopy. The percentage of parasitized erythrocytes (PPE) was calculated from a minimum of 500 total erythrocytes. The plates were incubated at $37{ }^{\circ} \mathrm{C}$ in a humidified atmosphere of $5 \% \mathrm{CO}_{2}$, $2 \% \mathrm{O}_{2}$, and $93 \% \mathrm{~N}_{2}$. 


\section{B. duncani cultures initiated in $\mathrm{HL}-1$ medium from washed hamster iRBC}

Cultures were initiated from $B$. duncani washed infected RBC (iRBC) within $2 \mathrm{~h}$ of collection from hamster 1 . Duplicate wells received $25 \mu 1$ of $i R B C, 100 \mu l$ of normal hamster RBCs (TAMU hamster), and $900 \mu 1$ of HL-1 medium. Duplicate wells received $60 \mu \mathrm{l}$ iRBC, $60 \mu \mathrm{l}$ of normal hamster RBCs (TAMU hamster), and $900 \mu \mathrm{l}$ of HL-1 medium. One well received $125 \mu \mathrm{l}$ of infected RBCs and $900 \mu \mathrm{l}$ of HL1 medium. In addition, one well was initiated from hamster 1 iRBC stored at $4{ }^{\circ} \mathrm{C}$ for 13 days using $70 \mu \mathrm{l}$ inoculum, $70 \mu \mathrm{l}$ of normal hamster RBC (TAMU hamster), and $900 \mu \mathrm{HL}-1$ medium. Cultures were similarly initiated from hamster 2 washed $\mathrm{iRBC}$ after 10 days refrigeration as follows. Two wells received $70 \mu \mathrm{l}$ iRBC, $70 \mu \mathrm{l}$ of normal hamster RBC (TAMU hamster), and $900 \mu \mathrm{l}$ HL-1 medium.

Subcultures were performed at either a 1:2 or 1:5 split ratio, depending on the PPE and how rapidly the parasites were growing. Wells were subcultured every 2 to 7 days when the well PPE reached approximately $1.5-2$ during the 2 nd to 7 th subculture and 3.5-19 in later subcultures. After the medium was replenished, the cells were resuspended by gentle pipetting. For a 1:2 subculture, $600 \mu \mathrm{l}$ of the resuspended culture was added to a well containing $600 \mu \mathrm{l}$ medium and $50 \mu \mathrm{l}$ donor hamster RBC. For a 1:5 subculture, $250 \mu \mathrm{l}$ of the resuspended culture was added to a well containing $900 \mu \mathrm{lme}$ dium and $70 \mu \mathrm{l}$ donor hamster RBC.

\section{B. duncani cultures initiated in M199 medium from washed hamster iRBC}

One culture well was initiated from hamster 1 washed $\mathrm{iRBC}$ stored at $4{ }^{\circ} \mathrm{C}$ for less than $48 \mathrm{~h}$, and another was initiated from the same stored $\mathrm{iRBC}$ after $13 \mathrm{~d}$ at $4{ }^{\circ} \mathrm{C}$. Two culture wells were initiated from hamster $2 \mathrm{iRBC}$ within $2 \mathrm{~h}$ of collection. Each well received $70 \mu \mathrm{l}$ iRBC, $70 \mu \mathrm{l}$ of normal hamster RBC (TAMU hamster), and $900 \mu 1$ M199 medium (as described above).

Two additional culture wells were initiated in M199 medium from existing $B$. duncani cultured in HL-1 medium. One well was subcultured from a $B$. duncani initial culture at a 1:2 split ratio and one well was subcultured from passage 3 $B$. duncani at a 1:5 split ratio. The cultures were maintained and monitored as described above.

\section{B. duncani cultures initiated in $\mathrm{HL}-1$ medium and non-hamster iRBC}

Culture wells were made using 60 or $100 \mu \mathrm{l}$ washed donor cow RBC and 60 or $25 \mu \mathrm{l}$ hamster $1 \mathrm{iRBC}$, respectively, and HL-1 medium for a total volume of $1.25 \mathrm{ml}$. Horse RBC $(100 \mu \mathrm{l})$ were added to culture wells containing $25 \mu \mathrm{l}$ hamster
$1 \mathrm{iRBC}$ and $900 \mu \mathrm{HL}-1$ medium. In addition, B. duncani culture $(250 \mu \mathrm{l})$ in hamster RBC was subcultured 1:5 into wells containing $70 \mu \mathrm{l}$ donor washed RBC from cow, horse, rat, or mouse and $900 \mu \mathrm{l}$ HL-1 medium. The cultures were maintained and monitored as described above.

\section{Cryopreservation}

Cultures of B. duncani were cryopreserved at a PPE of 6 or greater. The selected cultures were resuspended, collected, and centrifuged at $870 \times \mathrm{g}$ for $3 \mathrm{~min}$ in a microfuge to pellet the cells, and then the supernatant was removed. Cold PSG+G with 20\% PVP-40 (Sigma, St. Louis, MO) as the cryoprotectant was added in equal volume to the cell pellet. The cells were gently resuspended and $0.25-0.5 \mathrm{ml}$ was dispensed into each pre-chilled cryovial. The vials were placed in a $-80{ }^{\circ} \mathrm{C}$ ethanol bath overnight and then transferred to liquid nitrogen storage.

\section{Revival from cryopreservation in $\mathrm{HL}-1$}

Cryovials of cultured B. duncani piroplasms (A1) were removed from liquid nitrogen storage and the contents thawed rapidly by swirling the vials in a $37^{\circ} \mathrm{C}$ water bath. Cryovials were from passages 5,7 , and 12 and had been cryopreserved and stored in liquid nitrogen for 53 days, 44 days, and 26 days, respectively. The thawed contents were dispensed in $250 \mu \mathrm{l}$ aliquots into wells prepared with $900 \mu \mathrm{HL}-1$ medium and $100 \mu \mathrm{l}$ of washed donor hamster RBC. The plate was incubated at $37{ }^{\circ} \mathrm{C}$ in a humidified atmosphere of $5 \% \mathrm{CO}_{2}, 2 \% \mathrm{O}_{2}$, and $93 \% \mathrm{~N}_{2}$. The cultures were given fresh medium daily and monitored by Giemsa-stained blood film examination as described above.

\section{Images}

Figure 2 was captured using an Olympus BX41 microscope (Olympus America, Inc. Center Valley, PA) equipped with SpotSoftware 5.2 Macro-Photography(C 2016 SPOT Imaging (Diagnostic Instruments, Inc. Avon, MA).

\section{Results}

\section{In vivo}

\section{Hamsters inoculated for culture}

After inoculation with the WA1 inoculum (BEI \#NR-12311), hamster 1 reached a PPE of 13 on day 10 post-inoculation (p.i.) and blood was collected. Hamster 2 was inoculated with whole blood from hamster 1 and on day 3 p.i., the PPE was 10 . Results are summarized in Table 1. 
Fig. 2 B. duncani piroplasms from day 1 initial culture using HL-1 medium and hamster RBC. Several parasite forms are shown. (A) Early ring. (B) Early tetrad. (C) Mature ring. (D) Mature tetrad. Giemsa stain, $\times 1000$ oil immersion

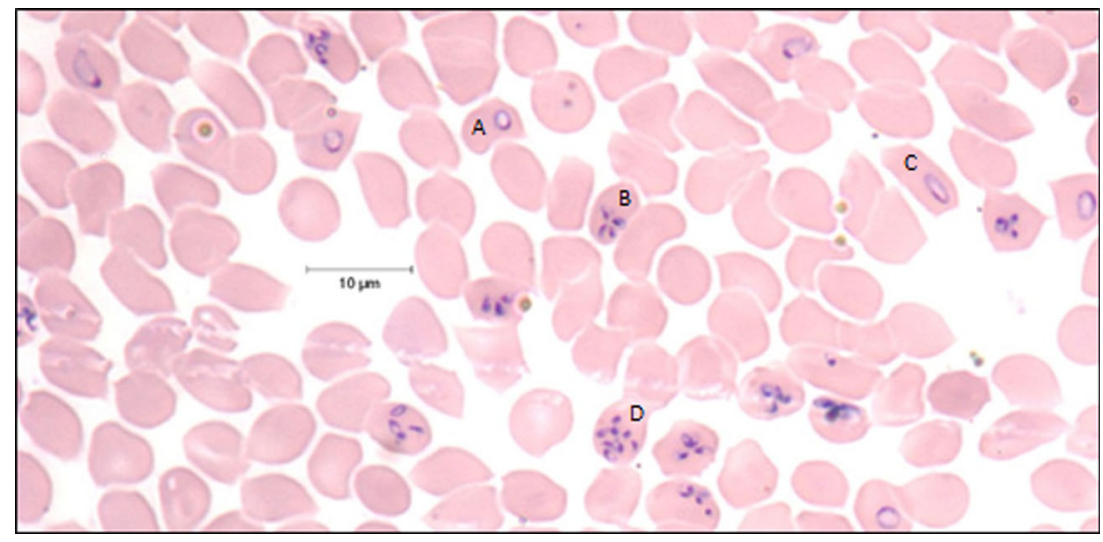

\section{Hamster inoculated to test the infectivity of cultured B. duncani}

Cryopreserved P25 B. duncani culture (cultivated for 4 months prior to cryopreservation and then stored in liquid nitrogen for 34 days) was inoculated i.p. into hamster 3. PPE observed in blood smears obtained from hamster 3 followed the same timeframe as hamster 1 (Table 1) and both were humanely euthanized at day 10 post-inoculation when PPE exceeded 10 . The only clinical signs observed in any of the inoculated hamsters were lethargy at peak parasitemia at time of terminal blood collection for hamsters 1 and 3 .

\section{In vitro}

\section{B. duncani cultures initiated from BEl cryostock in HL-1 and M199 media}

The cultures initiated directly from the BEI cryostock of B. duncani-infected hamster blood showed severe hemolysis in both HL-1 and M199 media on day 1 after inoculation. Many extracellular parasites were seen upon examination of the daily RBC smears. A few rare (0-4 iRBC/500 $\mathrm{RBC}$ ) early ring forms as observed in Abraham et al. (2018) were seen each day until day 14. An average of 2 infected blood cells out of 1000 were seen on day 16 postinoculation. The wells were subcultured 1:5 into single new wells for each medium. No parasites were seen after day 16 post-subculture in the new subculture wells or in the original wells (data not shown).

\section{Cultures initiated in HL-1 medium from washed $B$. duncani iRBC}

B. duncani parasitized erythrocytes and many extracellular parasites were evident within $24 \mathrm{~h}$ in cultures initiated from hamster 1 iRBC in HL-1 medium. One culture initiated with $25 \mu$ of infected hamster RBC was subcultured on day 2 at a 1:5 split ratio. This line (A1) continued to thrive with passages 2,3 , and 4 of this subcultured line effected on day 8,15 , and 20 , respectively. Later passages were done at similar time intervals as shown in Fig. 4. Throughout the propagation of this line, both the original well and the subcultured well were continuously maintained. Cultured parasites (Fig. 2) appeared identical in morphology to $B$. duncani in vivo. Cultures were maintained for over 160 days by subculturing an average of every 4.2 days using donor hamster RBC (Fig. 3). Figure 4 shows the peak parasitemia reached at time of subculture ( $\square$ ). Over time, the variability of parasite growth decreased and the cultures consistently needed to be subcultured every 3-4 days during the last 20 of the 160 days shown in Fig. 4 by the diamond marker $\bullet$. Cultured $B$. duncani (A1) was deposited with BEI Resources and is available under NR-50440 Babesia duncani, WA1 (in vitro) (parasitic protozoa).

B. duncani cultures initiated with 60 or $125 \mu$ infected hamster $1 \mathrm{RBC}$ initially propagated similarly as above but were subcultured only once at days 5 and 2, respectively,
Table 1 Babesia duncani initial and final PPE from passaging through Syrian hamsters

\begin{tabular}{llllll}
\hline $\begin{array}{l}\text { Inoculated } \\
\text { hamster }\end{array}$ & $\begin{array}{l}\text { Inoculum } \\
\text { PPE }\end{array}$ & Inoculum & $\begin{array}{l}\text { Day parasites first } \\
\text { seen }\end{array}$ & $\begin{array}{l}\text { Day blood } \\
\text { collected }\end{array}$ & $\begin{array}{l}\text { PPE at } \\
\text { collection }\end{array}$ \\
\hline 1 & $10 \%$ & $\begin{array}{c}\text { Cryopreserved whole } \\
\text { blood }\end{array}$ & 6 & 10 & $13 \%$ \\
2 & $13 \%$ & $\begin{array}{c}\text { Hamster 1 whole } \\
\text { blood } \\
\text { Cryopreserved P25 } \\
\text { culture }\end{array}$ & 1 & 3 & $10 \%$ \\
\hline
\end{tabular}


Fig. 3 B. duncani growth in continuous culture over 160 days. Cultures were initiated from hamster $1 \mathrm{iRBC}$ and maintained in donor hamster RBC and HL-1 medium. On day 66 an exceptionally high of 28 was recorded and is indicated by an asterisk (*). PPE at subcultures are indicated ( $\square$ )

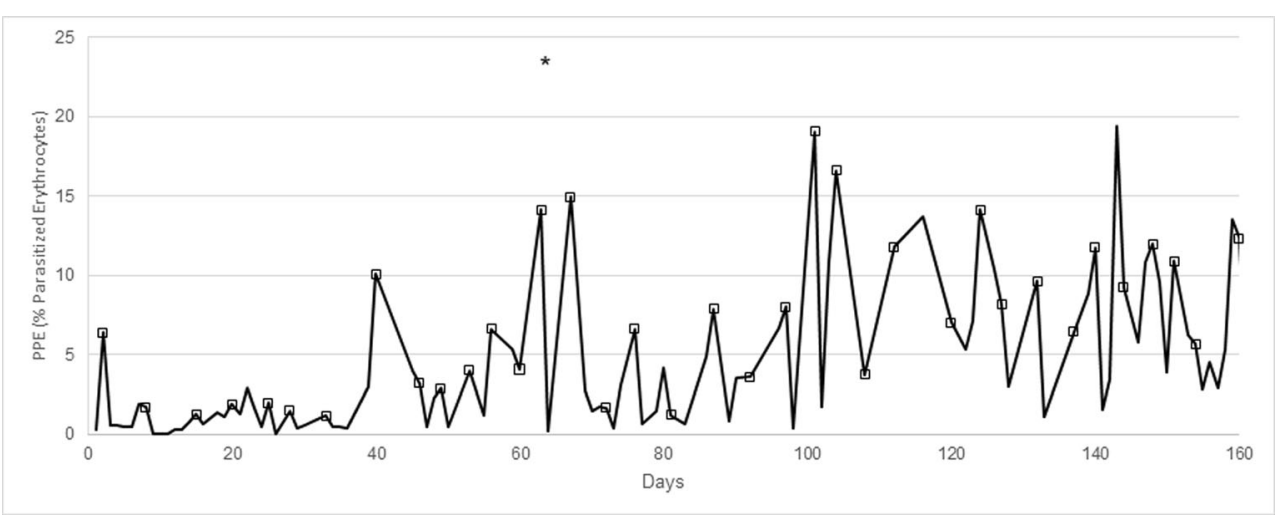

and then discontinued due to limited supply of hamster donor RBC (data not shown).

Two cultures initiated with $25 \mu$ of infected hamster 2 RBC in HL-1 medium were subcultured on days 2 and 4, respectively, when the PPE reached approximately 3 (Fig. 5). All wells were continuously maintained as described above. One culture became established whereas the other died out after 1 week as shown in Fig. 5.

Medium 199 did not continuously support B. duncani in vitro when initiated from either infected hamster 1 or hamster 2 blood, irrespective of whether fresh or stored inoculum was used. Two culture wells from hamster 2 in M199 underwent one subculture but PPE decreased thereafter until no viable parasites were visible on Giemsa-stained slides. Overall, parasites persisted in some M199 cultures for up to 2 weeks but none of the cultures became established (data not shown).

\section{B. duncani cultures initiated in $\mathrm{HL}-1$ medium and non-hamster iRBC}

B. duncani in hamster RBC, either blood derived or culture subpassage, were used as inocula in cultures that used normal donor RBC from cow, horse, rat, and mouse donor RBC. $B$. duncani in hamster RBC, either blood derived or culture subpassage, were used as inocula. Results for cow, horse, and rat are shown in Fig. 6. Mouse RBC cultures underwent severe hemolysis and excessive cellular debris obscured RBC on microscopy to the point that PPE could not be calculated. None of the alternate donor RBC was able to support in vitro cultivation of $B$. duncani. It was observed that after infected hamster RBC were introduced into culture with noticeably larger RBC, such as horse or cow, the parasites appeared to infect residual hamster RBC instead of invading different host RBC.

\section{Revival from cryopreservation in HL-1}

Cryopreserved culture from $B$. duncani $\mathrm{A} 1$ passages 5, 7, and 12 were successfully revived in HL-1 medium with hamster RBC. Free-floating parasites were visible the day after inoculation. Intracellular parasites became visible as early ring forms (Abraham et al. 2018) on stained smears relative to the passage level at which they were cryopreserved. Early ring forms began to be seen in the P12 revival at day 8 post-inoculation, $\mathrm{P} 7$ revival had a few at day 10 , and P5 revival showed visible early ring forms at day 16 . All revived cultures were maintained successfully for over 2 months (data not shown) at which point they were discontinued.

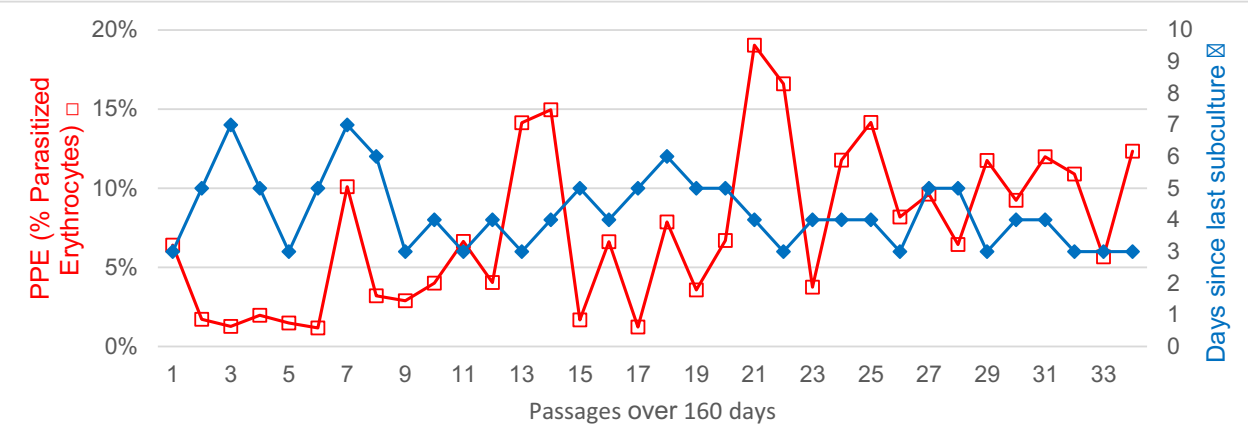

Fig. 4 B. duncani PPE at time of subculture over 35 passages and days between subcultures. Cultures were initiated from infected hamster 2 blood and maintained in donor hamster RBC and HL-1or M199 medium. PPE at subcultures are indicated ( $\square)$. Over time, variability of parasite growth decreased and the cultures consistently needed subcultured every 3-4 days during the last 20 of the 160 days shown in Fig. 4 by the diamond marker ( $\bullet)$ 
Fig. 5 B. duncani cultured in donor RBC from horse, cow, or rat using HL-1 medium. PPE on the day of subculture is indicated (口)

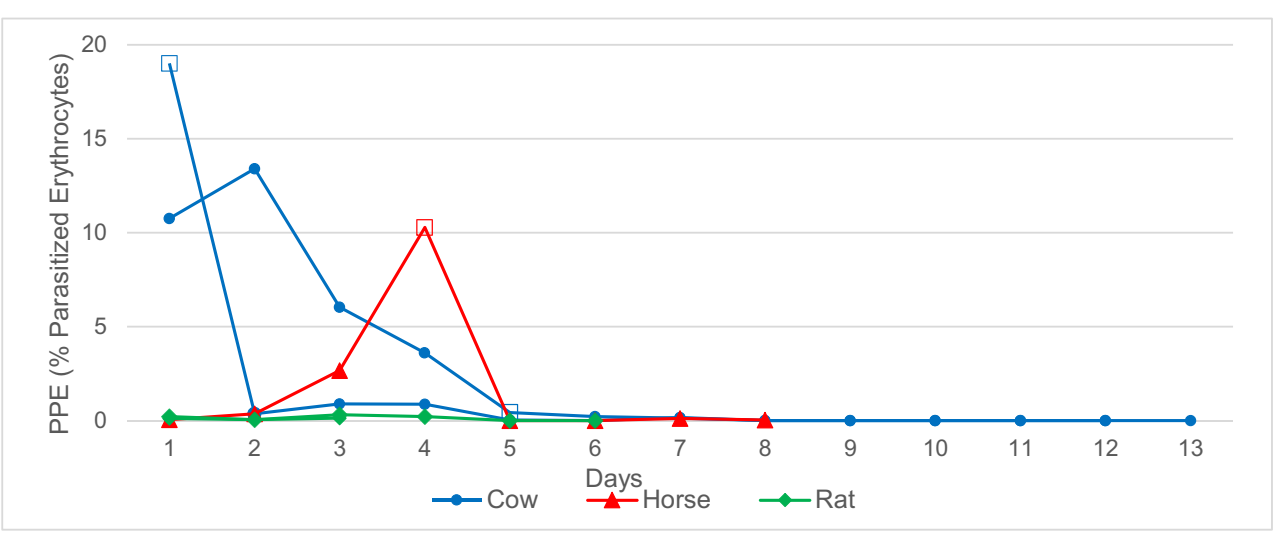

\section{Discussion}

B. duncani continuous cultures were successfully established when initiated using inoculum from infected Syrian hamsters. $B$. duncani remained viable for culture in washed erythrocytes from infected blood for at least 13 days when stored under refrigeration. Although $B$. duncani did not adapt to culture directly from infected hamster blood cryostock, the cryopreserved cultured parasites were successfully recovered both in vitro and in vivo from liquid nitrogen storage. Parasites cryopreserved in the 25 th culture passage were infective in vivo, with the hamster developing parasitemia similar to the course seen with the hamster inoculated with the BEI B. duncani-infected hamster blood cryostock (BEI \#NR12311). Certain Babesia spp. cultured over time shows decreased infectivity and as a result, some attenuated strains have been adapted as vaccines (Schuster 2002). While our current results showed no difference between the hamsters that received 25 th passage cultured parasites versus the BEI infected blood cryostock, it remains to be determined whether attenuation will occur as $B$. duncani undergoes more passages in vitro.

The hamsters inoculated with $B$. duncani in this study, regardless of source, showed a rapid increase in parasitemia from approximately 2 to $10 \%$ or greater within $24 \mathrm{~h}$. The hamsters were monitored for parasitemia early in the morning daily. This rapid increase in parasitemia may resemble the higher parasitemia seen in immunocompromised patients and other cases of severe disease from $B$. duncani.

One of the challenges to overcome when cultivating Babesia spp. continuous cultures is selecting an appropriate donor animal for routine blood supply (Alhaboubi et al. 2017). Even within the appropriate species, erythrocytes and/or serum from some animals may not be suitable for supporting the parasite in vitro and may inhibit parasite growth (Schuster 2002; Alhaboubi et al. 2017). It is possible immune factors such as antibodies or cytokines contribute to this difficulty even when red blood cells are washed and the buffy coat removed as thoroughly as feasible.

The Syrian hamster is the most frequently used animal model for human babesiosis, including B. microti and B. duncani (Souza et al. 2016). Hamster blood obtained from commercial sources is usually a pooled sample from multiple animals. To facilitate repeated blood collection from the same animals that supported the parasite, a small in-house colony of hamsters was maintained for blood donors. However, only a small quantity of hamster blood was retrieved per draw (approximately $1 \mathrm{ml}$ whole blood) in order to stay within humane limits. This impacted the number of cultures we could initiate and maintain. The need for repeated and larger blood collections encouraged our attempt to experiment with donor blood more readily available through tissue sharing, which included
Fig. 6 B. duncani cryostocks from P5, P7, and P12 were revived. PPE on the day of subculture is indicated $(\square)$

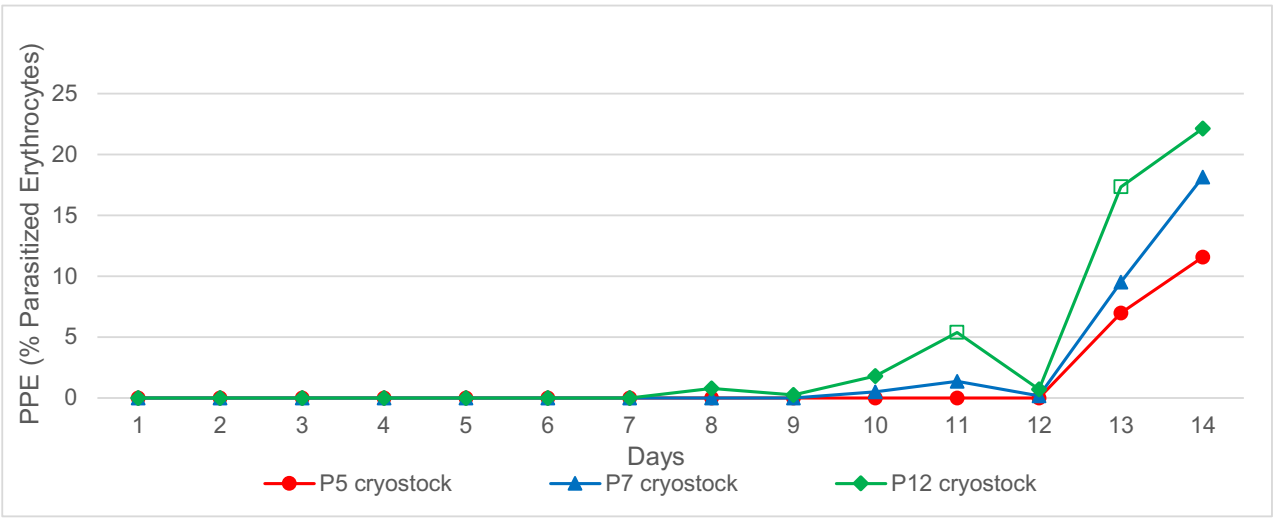


horse, cow, rat, and mouse. However, hamster RBC were the only donor RBC that supported parasite propagation and resulted in the establishment of $B$. duncani continuous cultures.

In the current study, Medium 199 as previously described for B. duncani in vitro culture (Thomford et al. 1994) was compared with HL-1 medium. In our hands, M199 did not support the parasite despite the reported earlier success with PPE consistently peaking at 20 to 30 every 2 to 3 days (Thomford et al. 1994). The reason for this disparity is unknown but possibly may be due more to the fetal bovine serum or the donor hamster RBC used in the different studies rather than to the medium composition. The M199supplemented medium is composed of $40 \%$ fetal bovine serum whereas HL-1, which is formulated as an enriched serumfree medium for hybridoma culture, is supplemented with only $20 \%$. The enriched formulation of HL-1with additional supplementation seems to have provided the required nutrients to allow the establishment of continuous $B$. duncani cultures in this study. Another more likely theory is that the high parasitemia collected from infected hamsters led to a slower initial growth rate as observed by Abraham et al. (2018). The growth curve did become more consistent and increase PPE over time (Figs. 3 and 4); however, it did not reach the consistent 20 to $30 \%$ PPE every 2 to 3 days as Thomford et al. (1994) initially experienced.

Although lower parasitemias resulted in the current cultures, the cultured parasites were robust and the stocks submitted to BEI were expanded to provide a source of cultured B. duncani to the scientific community. The utility of our cultured line is demonstrated in the work of Abraham et al. who tested therapeutic anti-babesial drug efficacy in vitro (2018). They tested four currently recommended treatments for human babesiosis (atovaquone, azithromycin, clindamycin, and quinine), and the results suggest these therapies have limited efficacy in cases of human babesiosis caused by $B$. duncani infection. B. duncani cultures in hamster RBC established in this current study were utilized for the establishment of the continuous culture in human RBC. As such, the hamster RBC growth medium (HaRGM) is nearly identical to the supplemented HL-1 medium used in this study except the addition of $100 \mu \mathrm{g}$ of gentamicin. Other species of Babesia have also been cultivated using a similar medium (Holman et al. 1994; Alhaboubi et al. 2017). Modulatorincubator conditions (gas, humidity, temperature) and the modulator-incubator were the same as this study. The human RBC growth medium (HuRGM) from Abraham et al. differed from HaRGM by including $20 \%$ human serum type A+ instead of FBS and much higher L-glutamine concentration (200 mM).

Babesiosis remains a nationally notifiable condition and a high-risk blood supply contaminant. In 2015, the Blood Product Advisory Committee (BPAC) in the USA recommended nationwide testing of blood donations and the development of more sensitive molecular assays for Babesia spp. in states where babesiosis is endemic (Karkoska et al. 2017). Neither the USA nor Canada has in place federally licensed tests for screening donated blood. Federally licensed diagnostics are still needed in the USA and Canada for $B$. duncani blood donor testing and accurate patient diagnosis. The ability to culture Babesia spp. can speed this process by providing a continuous supply of sufficient pathogen to test.

Continuous cultures of $B$. duncani were successfully established in the current study and were shown to remain viable and infective after cryopreservation. $B$. duncani cultures were deposited with BEI Resources and cultured $B$. duncani is now offered as a resource to other researchers. Cultured $B$. duncani provides a continuous laboratory resource available for molecular and biochemical studies, antibabesial drug testing, diagnostic tests, and vaccine development (Canning and Winger 1987; Schuster 2002; Alhaboubi et al. 2017; Abraham et al. 2018). It also reduces the overall use of animals in research by providing an alternative to maintaining a source of parasites by passaging through the animal host. As a result of this study, now one of the causes of human babesiosis, $B$. duncani, is available to the scientific community as a cryostock that can be directly cultured in vitro. The line described herein was utilized by Abraham et al. (2018) to adapt $B$. duncani to continuous culture in human RBC for their research into effective clinical therapies.

\section{Compliance with ethical standards}

Ethical approval All applicable international, national, and/or institutional guidelines for the care and use of animals were followed. All procedures performed in studies involving animals were in accordance with the ethical standards of Texas A\&M University at which the studies were conducted.

Conflict of interest The authors declare that they have no conflict of interest.

\section{References}

Alhaboubi A, He L, McCormack K, Gustafson A, Holman PJ (2017) Recovery of bovine Babesia spp. after long-term cryostorage and comparison of bovine donor erythrocytes and serum. Vet Parasitol 243:109-114. https://doi.org/10.1016/j.vetpar.2017.06.017

Abraham A, Brasov I, Thekkiniath J, Kilian N, Lawres L, Gao R, DeBus K, He L, Yu X, Zhu G, Graham M, Liu X, Molestina R, Ben Mamoun C (2018) Establishment of a continuous in vitro culture of Babesia duncani in human erythrocytes reveals unusually high tolerance to recommended therapies. J Biol Chem 293(52):19974 19981. https://doi.org/10.1074/jbc.AC118.005771

Canning EU, Winger CM (1987) Babesiidae.In: Taylor AER, Baker JR (Eds.) In vitro methods for parasite cultivation. Academic Press, London, pp199-299

Centers for Disease Control and Prevention (CDC) (2015) 2013 Data archive. https://www.cdc.gov/parasites/babesiosis/data-statistics/ 2013.html. Accessed 7 Nov 2018 
Centers for Disease Control and Prevention (CDC) (2016) 2012 Data archive. https://www.cdc.gov/parasites/babesiosis/data-statistics/ 2012.html. Accessed 7 Nov 2018

Conrad PA, Kjemtrup AM, Carreno RA, Thomford J, Wainwright K, Eberhard M, Quick R, Telford SR, Herwaldt B (2006) Description of Babesia duncani n.sp. (Apicomplexa: Babesiidae) from humans and its differentiation from other piroplasms. Int J Parasitol 36(7): 779-789. https://doi.org/10.1016/j.ijpara.2006.03.008

Herwaldt BL, Montgomery S, Woodhall D, Bosserman EA (2012) Babesiosis surveillance -18 states, 2011. CDC Morbidity and Mortality Weekly Report 61(27):505-509 https://www.cdc.gov/ $\mathrm{mmwr} /$ preview/mmwrhtml/mm6127a2.htm. Accessed 7 Nov 2018

Herwaldt BL, Gray EB (2016) Surveillance for Babesiosis - United States, 2014 annual summary. https://www.cdc.gov/parasites/ babesiosis/resources/babesiosis_surveillance_summary_2016.pdf. Atlanta, Georgia: U.S. Department of Health and Human Services, CDC. Accessed 7 November 2018

Holman PJ, Chieves L, Frerichs WM, Olson D, Wagner GG (1994) Babesia equi erythrocytic stage continuously cultured in an enriched medium. J Parasitol 80:232-236. https://doi.org/10.2307/3283752

Innes RJ (2013) Odocoileus hemionus. In: Fire effects information system, [online]. U.S. Department of Agriculture, Forest Service, Rocky Mountain Research Station, Fire Sciences Laboratory (Producer). https://www.fs.fed.us/database/feis/animals/mammal/ odhe/all.html. Accessed 8 November 2018

Karkoska K, Louie J, Appiah-Kubi AO, Wolfe L, Rubin L, Rajan S, Aygun B (2017) Transfusion-transmitted babesiosis leading to severe hemolysis in two patients with sickle cell anemia. Pediatr Blood Cancer 65:e26734. https://doi.org/10.1002/pbc.26734

Kjemtrup AM, Conrad PA (2000) Human babesiosis: an emerging tickborne disease. Int J Parasitol 30:1323-1337. https://doi.org/10.1016/ S0020-7519(00)00137-5

Lobo CA, Cursino-Santos JR, Alhassan A, Rodrigues M (2013) Babesia: an emerging infectious threat in transfusion medicine. PLoS Pathog 9(7):1-3. https://doi.org/10.1371/journal.ppat.1003387

Moritz ED, Winton CS, Tonnetti L, Townsend RL, Berardi VP, Hewins M, Weeks KE, Dodd RY, Stramer SL (2016) Screening for Babesia microti in the US blood supply. N Engl J Med 375:2236-2245. https://doi.org/10.1056/NEJMoa1600897

O'Connor KE, Kjemtrup AM, Conrad PA, Swei A (2018) An improved PCR protocol for detection of Babesia duncani in wildlife and vector samples. J Parasitol 104(4):429-432. https://doi.org/10.1645/17155
Schuster FL (2002) Cultivation of Babesia and Babesia-like blood parasites: agents of an emerging zoonotic disease. Clin Microbiol Rev 15(3):365-373. https://doi.org/10.1128/cmr.15.3.365-373.2002

Souza SS, Bishop HS, Sprinkle P, Qvarnstrom Y (2016) Comparison of Babesia microti real-time polymerase chain reaction assays for confirmatory diagnosis of babesiosis. Am J Trop Med Hyg 95(6):14131416. https://doi.org/10.4269/ajtmh.16-0406

Swei A, O'Connor KE, Couper LI, Thekkiniath J, Conrad PA, Padgett KA, Burns J, Yoshimizu MH, Gonzales B, Munk B, Shirkey N, Konde L, Ben Mamoun C, Lane RS, Kjemtrup A (2019) Evidence for transmission of the zoonotic apicomplexan parasite Babesia duncani by the tick Dermacentor albipictus. Int Journ Parasitol 49:95-103. https://doi.org/10.1016/j.jpara.2018.07.002

Thomford JW, Conrad P, Telford SR, Mathiesen D, Bowman BH, Spielman A, Eberhard ML, Herwaldt BL, Quick RE, Persing DH (1994) Cultivation and phylogenetic characterization of a newly recognized human pathogenic protozoan. J Infect Dis 169(5): 1050-1056. https://doi.org/10.1093/infdis/169.5.1050

Texas A\&M University System (TAMUS) (2011) The tick app for Texas and the southern region. http://tickapp.tamu.edu/ticks/wintertick. html. Accessed 9 Nov 2018

United States Food and Drug Administration (USFDA) (2016) Fatalities reported to FDA following blood collection and transfusion: annual summary for fiscal year 2015. http://wayback.archive-it.org/7993/ $20171114162532 / \mathrm{https}$ ://www.fda.gov/downloads/ BiologicsBloodVaccines/SafetyAvailability/ReportaProblem/ TransfusionDonationFatalities/UCM518148.pdf. Accessed 7 Nov 2018

United States Food and Drug Administration (USFDA) (2017) Fatalities reported to FDA following blood collection and transfusion: annual summary for fiscal year 2016. https://www.fda.gov/downloads/ BiologicsBloodVaccines/SafetyAvailability/ReportaProblem/ TransfusionDonationFatalities/UCM598243.pdf. Accessed 7 Nov 2018

Publisher's note Springer Nature remains neutral with regard to jurisdictional claims in published maps and institutional affiliations. 